\title{
GENETIC AND AGRONOMIC EVALUATION OF RAINFED TOMATO (SOLANUM LYCOPERSICUM L.) ACCESSIONS IN IBADAN, NIGERIA
}

\author{
Dickson Junior NWOSU $^{1 *}$, Christopher NWADIKE ${ }^{2}$, Gloria AFOLAYAN ${ }^{3}$, \\ Sunday ALADELE ${ }^{3}$, Anthony Ugochukwu OKERE ${ }^{4}$, Peter OMONOMO ${ }^{5}$ \\ ${ }^{1}$ National Centre for Genetic Resources and Biotechnology (NACGRAB), North Centre Zone, \\ Badeggi - Nigeria \\ ${ }^{2}$ Forestry Research Institute of Nigeria (FRIN), Department of Agricultural Technology, Federal \\ College of Forestry, Jos - Nigeria \\ ${ }^{3}$ National Centre for Genetic Resources and Biotechnology (NACGRAB), Department of Plant \\ Genetic Resources, Ibadan - Nigeria \\ ${ }^{4}$ National Centre for Genetic Resources and Biotechnology (NACGRAB), Biotechnology Unit, \\ Ibadan - Nigeria \\ ${ }^{5}$ National Centre for Genetic Resources and Biotechnology (NACGRAB), North Centre Zone, \\ Badeggi - Nigeria \\ * Corresponding author. E-mail: dicksonnwosu@gmail.com
}

\begin{abstract}
The extent of genetic variability among 19 accessions of tomato was studied using genetic variability parameters. Four-week old seedlings were transplanted in a well levelled field with 0.6 x 0.6 spacing and replicated three times in randomized complete block design. High significant differences among the accessions for all attributes studied. Cluster analysis based on 37 agro-morphological attributes separated accessions into two distinct groups according to the fruit types. Values for genotypic and phenotypic coefficients of variation showed variability among the accessions. Correlation analysis showed fruit/plant is positively and significantly correlated to plant height, number of branches/plant and leaf length. Very high genetic advance and heritability estimates for leaf length, leaf width, days to flower, days to $50 \%$ flowering, number of fruits/plant, fruit length, fruit diameter, fruit weight and 1000 seed weight suggest simple inheritance system and thus amenability for these attributes to selection in tomato improvement.
\end{abstract}

Keywords: attributes, genetic advance, heritability, inheritance, tomato, variance.

\section{Introduction}

Tomato (Solanum lycopersicon L. syn. - Lycopersicon esculentum Mill., Lycopersicon lycopersicum (L.) Karsten ex Farw.), is one of the most important vegetable crops grown over the world because of its wider adaptability, high yielding potential and suitability for variety of uses in fresh as well as processed food industries. It is one of the most important vegetable crops grown in Nigeria and utilized in almost every household for preparation of several dishes. Tomato plays an important role in human nutrition by providing essential amino acids, vitamins and minerals [SAINJU \& al. 2003]. Its vitamin C content is particularly high [KANYOMEKA \& SHIVUTE, 2005]. It also contains lycopene, a very potent antioxidant that may be an important contributor to prevention of cancers [AGARWAL $\&$ RAO, 2000]. With production of over 150 million tons of fresh fruit on 3.7 million hectares tomato exceeds the production of all other crops, with the exception of the potato and sweet potato [FAOSTAT, 2010]. Production in Nigeria has more than doubled in the last 10 years 
with the production in 2001, amounting to about 879,000 tonnes [AKANBI \& OLUDEMI, 2003]. However, commercial tomato production in Nigeria relies mostly on exotic introductions. The production of which is essentially restricted to the Northern Guinea Savanna and the Sudan ecologies due to favourable climatic conditions, particularly high insolation and low relative humidity. In nearly three decades up to 2013 , no tomato variety has been released in Nigeria [NACGRAB \& NASC, 2013]. The need therefore, to explore the production capabilities and potentials of long forgotten indigenous land races and other ecotypes has never been more urgent.

The concept of heritability which specifies the proportion of the total variation among a species due to genetic components combined with genetic advance. These are good parameters for determining gene action involved in the inheritance of any trait and by extension help in deciding the best breeding method to apply for improving such trait. High heritability indicates less environmental influence in the observed variation [SONGSRI \& al. 2008; EID, 2009], while high heritability accompanied by high genetic advance is an indication of additive gene action for such trait, making it most amenable to selection [TAZEEN \& al. 2009]. Determining the variability of yield and yield related components will enable the plant researcher to deduce the extent of environmental influence on yield, considering that yield and its components are quantitative characters and are affected by the environment.

This study was carried out to determine the extent of genetic variation and agronomic evaluation among available tomato accessions with the specific objective to use suitable genetic parameters such as phenotypic and genotypic variances, phenotypic and genotypic coefficients of variation and genetic advance as a basis for future breeding work in tomato.

\section{Material and methods}

Nineteen (19) accessions of tomato held in National Genebank at the National Centre for Genetic Resources and Biotechnology (NACGRAB) were planted for 2013 rainy season. The accessions were first planted in nursery trays placed in a mesh house after relevant seed treatment with Mancozeb®. Top soil was used for planting. Seedlings were transplanted at four weeks after planting (WAP) to NACGRAB research field, Moor Plantation (224m, $\left.7^{\circ} 23^{`}, 3^{\circ} 50^{\circ}\right)$, Ibadan, Nigeria. Seedlings were transplanted unto a well ploughed, harrowed and levelled field. Inter and intra-row spacing was 0.6 x $0.6 \mathrm{~m}$. Each treatment accession was in single 6 meter row plot. Total field size was $33 \mathrm{~m} \times 12 \mathrm{~m}$. The treatments were replicated thrice and laid out in a Completely Randomized Block Design. Recommended cultural practices were followed and irrigation was employed in the month of August when there was no rainfall. Data were recorded from five pre-tagged plants of each treatment. Attributes measured and recorded using descriptors for Tomato (Solanum spp.) [IBPGR, 1997] included: Leaf type, inflorescence type, stem pigmentation, stem pubescence, predominant fruit shape, colour of immature fruit, colour of ripe fruit, plant height $(\mathrm{cm})$, number of branches per plant, leaf length $(\mathrm{cm})$, leaf width $(\mathrm{cm})$ number of leaflets, number of days to first flowering, number of days to first fifty per cent flowering, number of days to ripening of first fruit, number of days to maturity, number of fruits per inflorescence, number of fruits per pedicel number of fruits per plant, peduncle length $(\mathrm{cm})$, fruit length $(\mathrm{mm})$, fruit diameter (mm), weight per fruit $(\mathrm{g})$, weight of 1000 seeds $(\mathrm{g})$ and fruit yield per plant $(\mathrm{g})$. Quantitative data obtained were subjected to Analysis of variance and significance means 
were separated using Duncan multiple range test (DMRT) using PBTools [PBTOOLS, 2014]. Mean values were used to estimate Genotypic and Phenotypic coefficients of variation as by [SINGH \& CHAUDHURY, 1985]. The broad sense heritability and genetic advance were calculated as proposed by [JOHNSON \& al. 1955] and simple linear correlation coefficient was determined according to [SNEDECOR \& COCHRAN 1967]. A correlation matrix was drawn up using the linear correlation coefficients. UPGMA cluster analysis was used to construct a dendogram to ascertain the genetic relationships among the tomato accessions.

\section{Results and discussions}

\section{Growth parameters}

There were statistically significant differences among the accessions on traits analyzed (Table 1). However, accession NG/Mr/MAY/09/005 had higher mean plant height while variety NG/AA/SEP/09/045 recorded the least values. The mean number of branches of accessions showed that NG/SA/01/10/002 recorded highest mean values while NHGB/09/113 recorded the least value. The mean number of leaflet showed significant difference among the accessions, NG/MR/MAY/09/005 recorded the highest values while NHGB/09/114 recorded the least. NG/MR/MAY/09/005 had highest mean leaf length value while NG/AA/SEP/09/042 had the least value. NG/0E/MAY/09/019 had highest mean leaf width value while NG/RM/JAN/10/001 had the least value. Accessions NG/0E/MAY/09/019; NG/AA/SEP/09/050 and NG/AA/SEP/09/013 had highest mean pedicel length while accession L00170 recorded the least values.

\section{Nigeria.}

Yield and Yield Components of Different Tomato Accessions In Ibadan

Yield and Yield Components of the Different Tomato Accessions were statistically significant among the accessions (Table 2). However, accession NG/Mr/MAY/09/005 had least mean number of days to flowering while NG/AA/SEP/09/013 recorded the highest mean number of days to flowering. Least mean value of days to $50 \%$ flowering was recorded for NG/AA/SEP/09/037 while highest mean value was recorded for NG/AA/SEP/09/042. Accessions NG/AA/SEP/09/013 and NG/AA/SEP/09/044 recorded least mean value for number of fruit per pedicel per plant while NGHB/09/114 and NG/RM/JAN/10/001 recorded high mean values respectively. NG/0E/MAY/09/019 and NG/AA/SEP/09/013 recorded high mean values for fruit weight while NG/RM/JAN/10/001 and L00169 recorded least mean values. Highest mean value for fruit length was recorded by NG/AA/SEP/09/037 and the least mean value recorded by NG/RM/JAN/10/001. Highest mean value for fruit width was recorded by NG/AA/SEP/09/013 while NG/RM/JAN/10/001. Highest mean value for fruit per plant was recorded by NG/RM/JAN/10/001 and NG/01/MAY/09/019 recorded the least mean value for this trait. Mean highest number of days to maturity was recorded for NG/AA/SEP/09/045 while L00169 had the least mean values. NG/AA/SEP/09/050 had the highest mean value for days to first fruit ripening while L00169 had the least value. Highest mean value for fruit yield per plant was recorded for NG/SA/01/10/002 while L00169 recorded least mean value. NG/MR/MAY/09/005 recorded highest mean value for 1000 seed weight while NG/AA/SEP/09/050 recorded the least mean value. 


\section{Genetic diversity}

Genetic diversity analysis for the accessions based on morphological characteristics measured, revealed that genetic distance ranging from 0.30 to 0.49 (Fig. 1). In this study, the cluster analysis based on 37 agro-morphological attributes that separated accessions into two distinct groups at 0.32 coefficient, which were according to fruit types - into cherry and classic fruit groups corresponding to varietal types (Figure 1). Cluster 1 included five accessions all of which are from south western Nigeria, while cluster 2 had 14 varieties from South-West Nigeria, South-South Nigeria and Republic of Benin (Table 3). Thirty-two of the 37 attributes recorded had morphological variation in 19 tomato accessions studied. The 20 qualitative attributes had two to nine numbers of observable types. Eight attributes (40\%) had more than two types, of which fruit shape had the largest variation with six types (slightly flattened, flattened, cylindrical, rounded, ellipsoid and high rounded). There were no obvious differences for five attributes (leaf type, division of leaf blade, stem pigmentation, abscission layer and flower colour) among the accessions studied.

\section{Genetic variability}

Analysis of variance for the means of all the measured attributes showed significant differences $(\mathrm{P} \leq 0.001)$ among the accessions (Table 4$)$. Values of genotypic and phenotypic variances were lowest in peduncle length and highest in fruit yield per plant. Higher values of genotypic and phenotypic variances were observed respectively for plant height $(21.89$, $40.75)$, number of branches $(319.60,499.91)$, leaf length $(31.08,31.95)$, number of leaflets (203.41, 441.06), days to flower $(33.23,41.75)$, days to $50 \%$ flower $(86.43,105.79)$, days to fruit ripening $(21.27,36.65)$ fruit per plant $(4349.08,4826.12)$, fruit length $(144.05,149.06)$, fruit diameter $(144.09,160.49)$, weight per fruit $(672.56,772.6)$, day to maturity $(28.07$, $31.94)$ and fruit yield per plant $(775796.12,1049841.90)$. The genotypic coefficient of variation (GCV) ranged from 6.06 in days to fruit ripening to 118.51 in fruit per plant. Similarly, PCV ranged from 7.96 (days to fruit ripening) to 124.84 (fruit per plant).

\section{Estimates of broad sense heritability $\left(\mathrm{H}^{2} \mathbf{b}\right)$ and genetic advance}

Estimates of heritability in the broad sense were very high for leaf length (97\%), leaf width $(88 \%)$, days to flower $(80 \%)$, days to $50 \%$ flowering $(82 \%)$, fruit per plant $(90 \%)$, fruit length $(97 \%)$, fruit diameter (90\%), fruit weight (100\%) and 1000 seed weight (Table 3). Peduncle length (39\%), number of leaflets per plant (46\%) and number of days to fruit ripening $(58 \%)$ had low to moderate heritability (Table 5). Very high genetic advance and heritability estimates were recorded for leaf length, leaf width, days to flower, days to $50 \%$ flowering, fruit per plant, fruit length, fruit diameter, fruit weight and 1000 seed weight.

\section{Character association}

Fruit yield per plant is positively and significantly $(\mathrm{P}<0.05)$ correlated to plant height $(r=0.481)$, number of branches per plant $(r=0.471)$ and leaf length $(r=0.507)$. Positive and significant association of number of fruits per plant with number of fruit per inflorescence $(r=0.726)$ is an indication of increased number of fruits with increased number of fruit bearing inflorescence. Weight per fruit which is a function of fruit size had predictably positive and significant association $(r=-0.582)$ with fruit length and fruit diameter. In this work, number fruit per plant was negatively and significantly correlated with fruit diameter $(\mathrm{r}=0.582)$. Number of branches had a significantly positive relationship with plant height $(\mathrm{r}$ $=0.782)$ and number of leaflets per plant $(\mathrm{r}=0.861)$ while maintaining negative and 
DICKSON JUNIOR NWOSU \& al.

significant relationships with days to flower $(\mathrm{r}=-0.752)$, days to $50 \%$ flowering $(\mathrm{r}=-0.609)$, days to fruit ripening $(r=-0.499)$, and days to maturity $(r=-0.505)-$ Table 6 .

Table 1. Means of agronomic attributes of nineteen tomato accessions in Ibadan, Nigeria

\begin{tabular}{lcccccc}
\hline Accessions & $\begin{array}{c}\text { Plant } \\
\text { height } \\
\text { (cm) }\end{array}$ & $\begin{array}{c}\text { Number } \\
\text { of } \\
\text { branches }\end{array}$ & $\begin{array}{c}\text { No of } \\
\text { leaflet }\end{array}$ & $\begin{array}{c}\text { Leaf } \\
\text { length } \\
\text { (cm) }\end{array}$ & $\begin{array}{c}\text { Leaf } \\
\text { width } \\
\text { (cm) }\end{array}$ & $\begin{array}{c}\text { Pedicel } \\
\text { length } \\
\text { (cm) }\end{array}$ \\
\hline NG/SA/01/10/002 & 17.15 & 11.00 & 51.11 & 37.87 & 23.00 & 0.60 \\
NGHB/09/120 & 16.2 & 7.28 & 51.0 & 29.07 & 21.80 & 0.50 \\
NG/AA/SEP/09/045 & 5.90 & 6.00 & 25.0 & 27.60 & 20.47 & 0.53 \\
NHGB/09/113 & 7.81 & 4.34 & 18.67 & 27.60 & 20.47 & 0.53 \\
NG/AA/SEP/09/044 & 13.72 & 9.33 & 59.0 & 27.77 & 19.57 & 0.50 \\
L00170 & 14.48 & 6.27 & 31.0 & 33.00 & 20.10 & 0.33 \\
NG/OE/MAY/09/019 & 9.63 & 5.55 & 26.06 & 37.70 & 27.00 & 0.70 \\
NG/AA/SEP/09/050 & 13.0 & 7.00 & 19.0 & 27.60 & 20.57 & 0.70 \\
NG/SA/07/10/002 & 12.53 & 6.28 & 29.72 & 30.90 & 20.57 & 0.37 \\
NG/AA/SEP/09/040 & 12.05 & 6.00 & 37.12 & 23.27 & 18.17 & 0.37 \\
NG/MR/MAY/09/005 & 24.67 & 10.78 & 73.78 & 42.20 & 26.03 & 0.60 \\
NG/AA/SEP/09/037 & 22.29 & 8.55 & 56.55 & 30.97 & 20.27 & 0.50 \\
NG/RM/JAN/10/001 & 8.54 & 6.89 & 26.44 & 19.77 & 12.50 & 0.50 \\
NG/MR/MAY/09/006 & 17.95 & 6.77 & 40.89 & 32.20 & 21.07 & 0.50 \\
NGHB/09/114 & 6.14 & 4.55 & 18.55 & 26.00 & 17.10 & 0.42 \\
NG/AA/SEP/09/013 & 13.09 & 7.66 & 41.18 & 29.90 & 20.30 & 0.70 \\
NG/AA/SEP/09/042 & 6.91 & 5.06 & 20.89 & 19.30 & 13.7 & 0.40 \\
L00169 & 9.60 & 7.17 & 45.0 & 28.20 & 16.7 & 0.37 \\
NG/AA/SEP/09/053 & 18.01 & 8.67 & 63.56 & 34.37 & 25.10 & 0.50 \\
F test & $* * *$ & $* * *$ & $* * *$ & $* * *$ & $* * *$ & $*$ \\
MSE & 2.67 & 0.89 & 9.39 & 0.68 & 1.09 & 0.07 \\
H 2 & 0.78 & 0.80 & 0.72 & 0.99 & 0.91 & 0.66 \\
\hline
\end{tabular}

$*, * * *=$ significant at $5 \%$ and $0.1 \%$ probability levels respectively.

MSE $=$ Standard error of mean

$\mathrm{H}^{2}=$ Heritability 
GENETIC AND AGRONOMIC EVALUATION OF RAINFED TOMATO ...

Table 2. Means of yield and yield components of nineteen tomato accessions in Ibadan, Nigeria

\begin{tabular}{|c|c|c|c|c|c|c|c|c|c|c|c|}
\hline Accessions & $\begin{array}{c}\text { Number } \\
\text { of days to } \\
\text { flower }\end{array}$ & $\begin{array}{c}\text { Number } \\
\text { of days to } \\
50 \% \\
\text { flowering }\end{array}$ & $\begin{array}{c}\text { Number } \\
\text { of fruits } \\
\text { per } \\
\text { pedicel }\end{array}$ & $\begin{array}{c}\text { Weight } \\
\text { per } \\
\text { fruit }(\mathrm{g})\end{array}$ & $\begin{array}{c}\text { Fruit } \\
\text { length } \\
(\mathrm{cm})\end{array}$ & $\begin{array}{c}\text { Fruit } \\
\text { width } \\
(\mathrm{cm})\end{array}$ & $\begin{array}{c}\text { Number } \\
\text { of fruits } \\
\text { per plant }\end{array}$ & $\begin{array}{c}\text { No of } \\
\text { days to } \\
\text { maturity }\end{array}$ & $\begin{array}{c}\text { No of } \\
\text { days to } \\
\text { ripening } \\
\text { of first } \\
\text { fruit }\end{array}$ & $\begin{array}{c}\text { Fruit } \\
\text { yield per } \\
\text { plant } \\
\text { (kg) }\end{array}$ & $\begin{array}{c}1000 \text { seed } \\
\text { weight } \\
\text { (g) }\end{array}$ \\
\hline NG/SA/01/10/002 & 39.00 & 43.00 & 5.67 & 47.92 & 37.7 & 45.3 & 79.7 & 73.0 & 68 & 3.81 & 1.593 \\
\hline $\mathrm{NGHB} / 09 / 120$ & 40.00 & 46.67 & 3.33 & 64.14 & 47.0 & 54.0 & 17.7 & 78.0 & 75.33 & 1.21 & 2.673 \\
\hline NG/AA/SEP/09/045 & 51.00 & 53.33 & 6.0 & 27.28 & 51.7 & 27.3 & 31.0 & 87.7 & 83.67 & 0.85 & 2.443 \\
\hline NHGB/09/113 & 45.00 & 47.00 & 3.33 & 52.72 & 33.3 & 47.0 & 19.3 & 80.0 & 64.67 & 1.05 & 2.140 \\
\hline NG/AA/SEP/09/044 & 42.00 & 43.00 & 3.0 & 11.86 & 22.0 & 33.3 & 10.0 & 78.0 & 78.67 & 1.13 & 2.073 \\
\hline L00170 & 44.67 & 46.67 & 6.67 & 10.13 & 24.0 & 26.7 & 78.3 & 80.0 & 75.67 & 1.15 & 2.563 \\
\hline NG/OE/MAY/09/019 & 46.00 & 50.67 & 4.67 & 101.8 & 49.0 & 57.0 & 11.0 & 86.0 & 82.00 & 1.12 & 2.446 \\
\hline NG/AA/SEP/09/050 & 49.00 & 53.00 & 4.0 & 27.28 & 34.3 & 35.5 & 7.0 & 87.3 & 85 & 0.19 & 1.123 \\
\hline NG/SA/07/10/002 & 49.00 & 48.00 & 5.0 & 28.12 & 32.3 & 40.0 & 33.7 & 86.0 & 84 & 0.90 & 2.806 \\
\hline NG/AA/SEP/09/040 & 43.67 & 46.67 & 4.67 & 43.12 & 35.0 & 45.0 & 52.7 & 83.0 & 74 & 2.30 & 2.960 \\
\hline NG/MR/MAY/09/005 & 29.67 & 43.33 & 5.0 & 46.36 & 41.3 & 43.3 & 52.0 & 77.7 & 71.67 & 2.41 & 2.967 \\
\hline NG/AA/SEP/09/037 & 34.00 & 41.00 & 5.0 & 27.26 & 59.3 & 32.7 & 81.7 & 74.3 & 71.00 & 2.21 & 2.617 \\
\hline NG/RM/JAN/10/001 & 39.00 & 43.33 & 8.33 & 2.92 & 14.0 & 14,7 & 305.0 & 75.0 & 74.67 & 0.58 & 2.447 \\
\hline NG/MR/MAY/09/006 & 43.00 & 49.67 & 6.0 & 25.20 & 29.7 & 42.0 & 53.0 & 74.7 & 72.33 & 1.29 & 3.335 \\
\hline $\mathrm{NGHB} / 09 / 114$ & 51.33 & 68.33 & 7.0 & 48.51 & 40.7 & 42.0 & 39.0 & 81.0 & 79.67 & 1.30 & 2.123 \\
\hline NG/AA/SEP/09/013 & 55.67 & 43.00 & 3.0 & 86.33 & 42.3 & 58.5 & 17.7 & 75.0 & 73.00 & 1.52 & 2.207 \\
\hline NG/AA/SEP/09/042 & 46.00 & 80.00 & 5.0 & 19.18 & 27.0 & 28.5 & 14.0 & 78.0 & 75.33 & 0.27 & 2.120 \\
\hline L00169 & 41.67 & 46.00 & 6.33 & 2.72 & 16.7 & 17.0 & 80.0 & 71.7 & 63.67 & 0.21 & 1.153 \\
\hline NG/AA/SEP/09/053 & 32.67 & 42.67 & 5.67 & 25.13 & 46.3 & 32.3 & 41.3 & 79.7 & 71.00 & 1.01 & 1.623 \\
\hline F test & $*$ & $* * *$ & $* * *$ & $* * *$ & $* * *$ & $* * *$ & $* * *$ & $* * *$ & $* * *$ & $* * *$ & $* * *$ \\
\hline MSE & 3.96 & 2.56 & 0.439 & 5.708 & 1.26 & 2.288 & 9.694 & 1.749 & 3.06 & 0.329 & 0.0076 \\
\hline $\mathrm{H}^{2}$ & 0.65 & 0.93 & 0.92 & 0.95 & 0.99 & 0.96 & 0.98 & 0.88 & 0.75 & 0.88 & 0.98 \\
\hline
\end{tabular}

$*, * *, * * *=$ significant at $5 \%, 1 \%$ and $0.1 \%$ probability levels respectively. MSE = Standard error of mean. $\mathrm{H}^{2}=\mathrm{Heritability}^{2}$ 


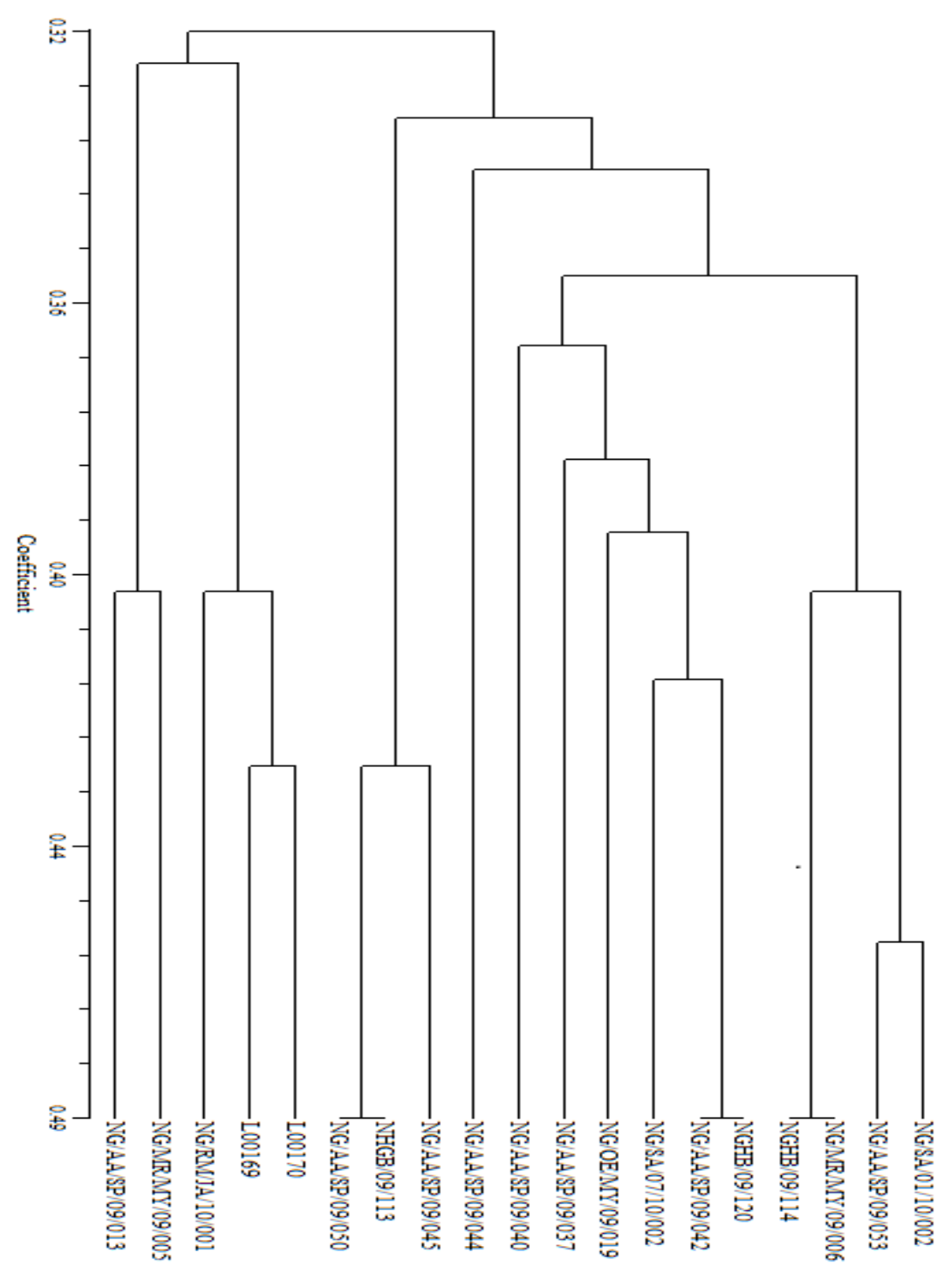

Figure 1. Dendogram of 19 tomato accessions based on 37 agro-morphological attributes and generated from average taxonomic distance matrix by UPGMA in NYSYSpc 


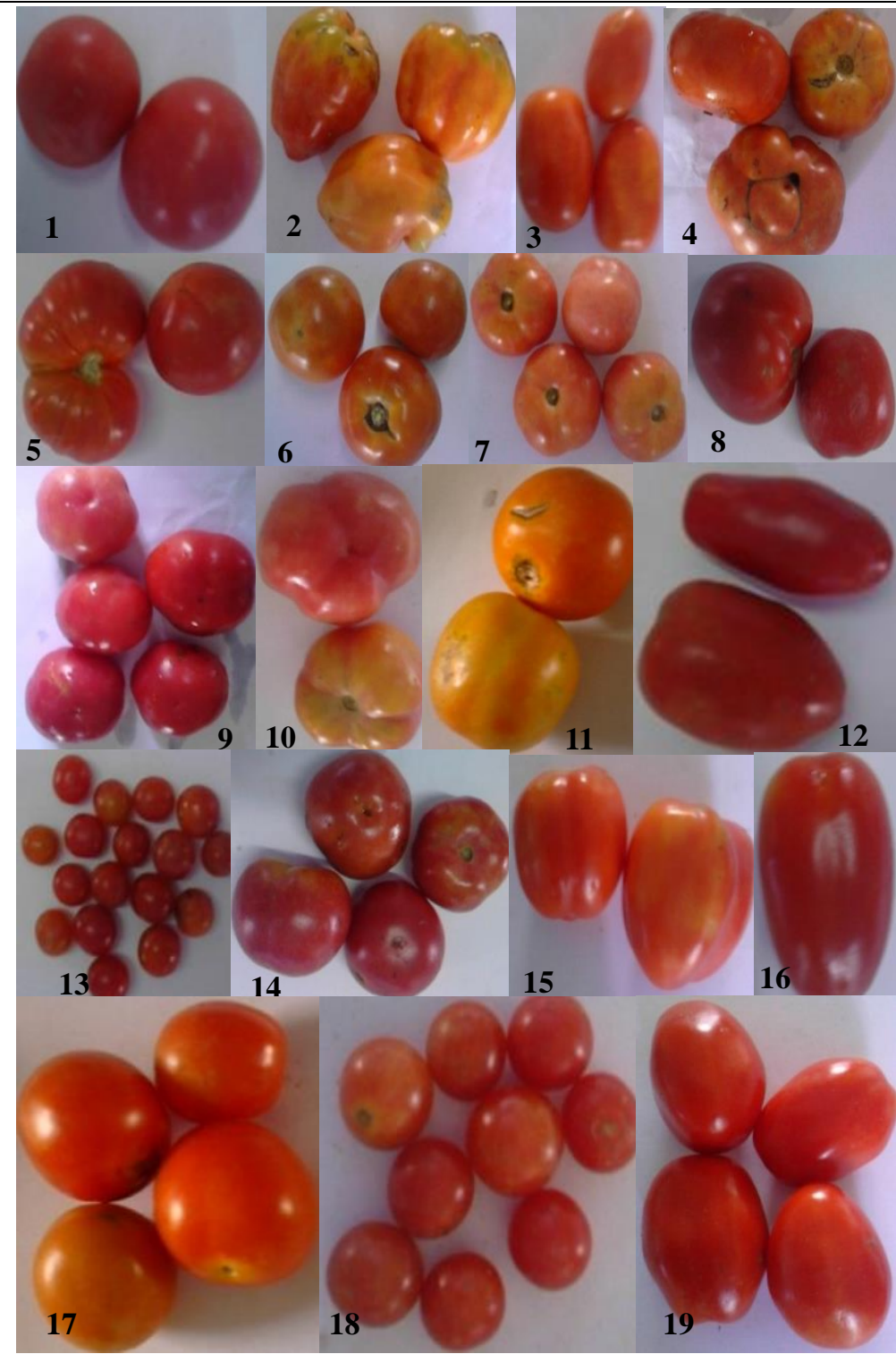

Figure 2. Genetic diversity as exhibited in fruits of 19 tomato accessions used for this study 1 - NG/SA/01/10/002, 2 - NGHB/09/120, 3 - NG/AA/SEP/09/045, 4 - NHGB/09/113, 5 NG/AA/SEP/09/044. 6 - L00170, 7 - NG/OE/MAY/09/019, 8 - NG/AA/SEP/09/050, 9 NG/SA/07/10/002, 10 - NG/AA/SEP/09/040, 11 - NG/MR/MAY/09/005, 12 - NG/AA/SEP/09/037, 13 - NG/RM/JAN/10/001, 14 - NG/MR/MAY/09/006, 15 - NHGB/09/114, 16 - NG/AA/SEP/09/013, 17 - NG/AA/SEP/09/042, 18 - L00169 and 19 - NG/AA/SEP/09/053. 
DICKSON JUNIOR NWOSU \& al.

Table 3. Accession names and sources of accessions used for the study

\begin{tabular}{llll}
\hline S. No. & \multicolumn{1}{c}{ Accession ID } & \multicolumn{1}{c}{ Source } & \multicolumn{1}{c}{ Region, Country } \\
\hline 1 & NG/SA/01/10/002 & Quagbo market & Republic of Benin \\
2 & NGHB/09/120 & Agbo, Delta state & South-South, Nigeria \\
3 & NG/AA/SEP/09/045 & Igede, Ekiti state & South-West, Nigeria \\
4 & NHGB/09/113 & Agbo, Delta state & South-South, Nigeria \\
5 & NG/AA/SEP/09/044 & Igede, Ekiti state & South-West, Nigeria \\
6 & L00170 & Ido, Oyo state & South-West, Nigeria \\
7 & NG/OE/MAY/09/019 & Omi adio Oyo state & South-West, Nigeria \\
8 & NG/AA/SEP/09/050 & Ijero ekiti, Ekiti state & South-West, Nigeria \\
9 & NG/SA/07/10/002 & Quagbo market & Republic of Benin \\
10 & NG/AA/SEP/09/040 & Ika ejigbo, Osun state & South-West, Nigeria \\
11 & NG/MR/MAY/09/005 & Osiele, Ogun state & South-West, Nigeria \\
12 & NG/AA/SEP/09/037 & Osun state & South-West, Nigeria \\
13 & NG/RM/JAN/10/001 & Ido, Oyo state & South-West, Nigeria \\
14 & NG/MR/MAY/09/006 & Omida, Ogun state & South-West, Nigeria \\
15 & NHGB/09/114 & Sapele, Delta state & South-South, Nigeria \\
16 & NG/AA/SEP/09/013 & Osun state & South-West, Nigeria \\
17 & NG/AA/SEP/09/042 & Iloko ijesa, Osun state & South-West, Nigeria \\
18 & L00169 & Ido, Oyo state & South-West, Nigeria \\
19 & NG/AA/SEP/09/053 & Oja oba ado, Ekiti state & South-West, Nigeria \\
\hline
\end{tabular}

Table 4. Analysis of variance for different characters in tomato accessions

\begin{tabular}{lcccc}
\hline \multicolumn{1}{c}{ Attribute } & $\begin{array}{c}\text { Accession } \\
\text { mean }\end{array}$ & F - value & $\begin{array}{c}\text { P-value } \\
\leq\end{array}$ & $\begin{array}{c}\text { Coefficient of } \\
\text { variation }\end{array}$ \\
\hline Weight/fruit (g) & 36.74 & 21.26 & 0.001 & 27.16 \\
1000 Seed weight (g) & 2.29 & 16753.0 & 0.001 & 0.35 \\
No of days to flowering & 42.40 & 12.69 & 0.001 & 12.98 \\
No of days to 50\% flowering & 49.40 & 14.39 & 0.001 & 8.79 \\
No of days to fruit ripening & 76.04 & 5.15 & 0.001 & 5.16 \\
No of days to fruit maturity & 78.65 & 20.96 & 0.001 & 2.54 \\
Plant height (cm) & 13.14 & 4.48 & 0.001 & 33.05 \\
Number of leaflets & 38.66 & 3.57 & 0.001 & 39.87 \\
Number of branches & 7.11 & 4.38 & 0.001 & 14.06 \\
Peduncle length (cm) & 0.51 & 2.95 & 0.05 & 22.97 \\
No of fruit/inflorescence & 5.14 & 11.79 & 0.001 & 14.06 \\
Fruit length (mm) & 35.98 & 87.20 & 0.001 & 6.22 \\
Fruit diameter (mm) & 38.01 & 27.35 & 0.001 & 10.66 \\
No of fruit/peduncle & 55.69 & 28.35 & 0.001 & 39.25 \\
Leaf length (cm) & 29.63 & 108.36 & 0.001 & 3.15 \\
Leaf width (cm) & 20.07 & 22.02 & 0.001 & 6.49 \\
Fruit yield per plant $(\mathrm{g})$ & 1269.64 & 15.75 & 0.001 & 2.54 \\
& & & & \\
\hline
\end{tabular}


Table 5. Estimates of phenotypic variance $\left(\sigma^{2} \mathrm{p}\right)$, genotypic variance $\left(\sigma^{2} \mathrm{~g}\right)$, heritability $\left(\mathrm{H}^{2} \mathrm{~b}\right)$, genotypic and phenotypic coefficients of variability and genetic advance for various attributes of tomato accessions

\begin{tabular}{|c|c|c|c|c|c|c|c|}
\hline Attributes & $\sigma^{2} \mathbf{g}$ & $\sigma^{2} p$ & $\sigma^{2} e$ & $\begin{array}{l}\mathbf{H}^{2} \mathbf{b} \\
(\%)\end{array}$ & GCV & PCV & GA \\
\hline Plant height & 21.89 & 40.75 & 18.86 & 54 & 35.60 & 48.58 & 53.75 \\
\hline $\begin{array}{l}\text { No of } \\
\text { branches/plant }\end{array}$ & 319.60 & 499.91 & 180.31 & 64 & 17.68 & 22.11 & 29.12 \\
\hline Leaf length & 31.08 & 31.95 & 0.87 & 97 & 18.82 & 19.08 & 38.23 \\
\hline Leaf width & 11.89 & 13.55 & 1.70 & 88 & 17.18 & 18.37 & 33.11 \\
\hline $\begin{array}{l}\text { Number of } \\
\text { leaflets/plant }\end{array}$ & 203.41 & 441.06 & 237.64 & 46 & 38.90 & 57.29 & 54.43 \\
\hline $\begin{array}{l}\text { No of days to } \\
\text { flower }\end{array}$ & 33.23 & 41.75 & 8.53 & 80 & 13.59 & 15.24 & 24.98 \\
\hline $\begin{array}{l}\text { No of days to } 50 \% \\
\text { flowering }\end{array}$ & 86.43 & 105.79 & 19.37 & 82 & 18.82 & 20.82 & 35.04 \\
\hline $\begin{array}{l}\text { No of days to fruit } \\
\text { ripening }\end{array}$ & 21.27 & 36.65 & 15.39 & 58 & 6.06 & 7.96 & 9.52 \\
\hline $\begin{array}{l}\text { No of } \\
\text { fruit/inflorescence }\end{array}$ & 1.88 & 2.40 & 0.52 & 78 & 26.67 & 30.15 & 48.60 \\
\hline No of fruit/plant & 4349.08 & 4826.12 & 477.04 & 90 & 118.51 & 124.84 & 231.74 \\
\hline Peduncle length & 0.01 & 0.02 & 0.01 & 39 & 18.54 & 29.53 & 23.98 \\
\hline Fruit length & 144.05 & 149.06 & 5.01 & 97 & 33.36 & 33.93 & 67.55 \\
\hline Fruit diameter & 144.09 & 160.49 & 16.40 & 90 & 31.58 & 33.33 & 61.64 \\
\hline Weight/Fruit & 672.56 & 772.6 & 99.6 & 100 & 75.58 & 75.63 & 135.69 \\
\hline Days to Maturity & 28.07 & 31.94 & 3.88 & 88 & 6.72 & 7.17 & 12.98 \\
\hline 1000 seed weight & 0.35 & 0.35 & 0.0 & 100 & 25.85 & 28.85 & 53.24 \\
\hline Fruit yield/plant & 775796.12 & 1049841.90 & 274045.48 & 74 & 63.37 & 80.70 & 122.85 \\
\hline
\end{tabular}

$\sigma^{2} \mathrm{~g}=$ genotypic variance, $\sigma^{2} \mathrm{~g}=$ phenotypic variance, $\mathrm{GCV}=$ genotypic coefficient of variation, $\mathrm{PCV}$ $=$ phenotypic coefficient of variation, $\mathrm{GA}=$ Genetic advance, $\mathrm{H}^{2} \mathrm{~b}=$ heritability in broad sense 
DICKSON JUNIOR NWOSU \& al.

Table 6. Genotypic (above) and phenotypic (below) correlation coefficients of vegetative and reproductive and yield attributes of 19 tomato accessions in

\begin{tabular}{|c|c|c|c|c|c|c|c|c|c|c|c|c|c|c|c|c|c|c|}
\hline \multicolumn{19}{|c|}{ Ibadan Nigeria } \\
\hline & NOL & NOB & PH & LL & LW & PDL & DTF & DTFF & $\begin{array}{c}\text { DTFF } \\
\text { Rp }\end{array}$ & DTM & FPI & WPF & FL & FD & FPP & FYPP & $\begin{array}{c}1000 \mathrm{~S} \\
\mathrm{~W}\end{array}$ & WtPS \\
\hline NOL & & $.960^{* * *}$ & $.875^{* *}$ & $.732 * *$ & $.579 * *$ & .149 & $-.998^{* * *}$ & $-.690 * *$ & -.400 & $-.541 *$ & -.226 & -.025 & .259 & .111 & .055 & .491 & .149 & .396 \\
\hline NOB & $.861^{* * *}$ & & $.804 * *$ & $.727 * *$ & $.462 *$ & .423 & $-.877^{* * *}$ & $-.682 * *$ & -.261 & $-.523 *$ & -.144 & -.073 & .135 & .011 & .106 & $.579 * *$ & -.131 & .283 \\
\hline PH & $.831^{* *}$ & $.780^{* * *}$ & & $.838 * *$ & $.628 * *$ & .281 & $-.998 * *$ & $-.639^{* * *}$ & -.248 & -.397 & -.196 & .031 & .352 & .223 & .062 & $.577 * *$ & .288 & $.488^{*}$ \\
\hline LL & $.630^{* * *}$ & $.653 * *$ & $.733^{* *}$ & & $.892 * *$ & .419 & $-.532 *$ & $-.520 *$ & -.218 & -.109 & -.122 & .293 & .388 & .368 & .255 & $.608 * *$ & .090 & $.620^{* * *}$ \\
\hline LW & $.469^{*}$ & .419 & $.558 *$ & $.854 * *$ & & $.675^{* *}$ & -.366 & $-.458 *$ & .065 & .289 & -.404 & $.594^{* * *}$ & $.636^{* * *}$ & $.619^{* *}$ & $.503^{*}$ & $.466^{*}$ & .086 & .831 ** \\
\hline PEDL & .085 & .313 & .189 & .336 & $.675 * *$ & & -.106 & -.297 & .177 & .155 & -.528 & $.739 * *$ & $.499 *$ & .614 & .221 & .220 & -.302 & $.510^{*}$ \\
\hline DTF & $-.751^{* *}$ & $-.637 * *$ & $-.743 * *$ & -.433 & -.366 & -.106 & & $.539 *$ & $.726^{* * *}$ & $.505^{*}$ & -209 & .323 & -.089 & .268 & .395 & $-.496 *$ & -.153 & -.257 \\
\hline DTFF & $-.587 * *$ & $-.589 * *$ & $-.563^{*}$ & $-.498^{*}$ & $-.458^{*}$ & -.297 & .539 & & .342 & .245 & .165 & -.029 & -.080 & -.096 & .278 & -.336 & -.119 & -.194 \\
\hline DTFFRp & -.406 & -.276 & -.276 & -.187 & .065 & .177 & $.726 * *$ & .342 & & $.908^{* *}$ & -.033 & .093 & .181 & .055 & .235 & -.355 & .075 & .124 \\
\hline DTM & $-.492^{*}$ & -.455 & -.359 & -.110 & .289 & .155 & $.505 * *$ & .245 & $.908 * *$ & & -.153 & .230 & .306 & .177 & .427. & -.317 & -.007 & .234 \\
\hline FPI & -.185 & -.103 & -.175 & -.113 & -.404 & $-.528 *$ & -.209 & .165 & -.033 & -.153 & & $-.542 *$ & -.316 & $-.678^{* *}$ & $.759^{* *}$ & .038 & .075 & -.297 \\
\hline WPF & -.041 & -.079 & .020 & .285 & $.594 * *$ & $.739 * *$ & .323 & -.029 & .093 & .230 & $-.542 * *$ & & $.601^{* * *}$ & $.925^{* *}$ & .455 & .371 & .135 & $.674 * *$ \\
\hline FL & .214 & .119 & .314 & .386 & $.636 * *$ & $.499 *$ & -.089 & -.080 & .181 & .306 & -.316 & $.601 * *$ & & $.535^{*}$ & .435 & $.466 *$ & .135 & $.996 * *$ \\
\hline FW & .075 & .013 & .197 & .360 & .619 ** & $.614^{* * *}$ & .268 & -.096 & .055 & .177 & $-.678^{* *}$ & $.925 * *$ & $.534^{*}$ & & $.584^{* * *}$ & $.455^{*}$ & .240 & $.614 * *$ \\
\hline FPP & -.021 & .114 & -.033 & -.250 & $-.503^{*}$ & -.221 & -.395 & -.278 & -.235 & -.427 & $.759^{* * *}$ & -.455 & -.435 & -.584 & & .018 & . 108 & $-.510^{*}$ \\
\hline FYPP & .408 & $.501 *$ & $.530^{*}$ & $.561 *$ & $.466^{*}$ & .219 & $-.496^{*}$ & -.336 & -.355 & -.317 & .038 & .371 & $.466^{*}$ & .045 & .018 & & .264 & $-.528 *$ \\
\hline SW1000 & .121 & -.114 & .255 & .089 & .086 & -.302 & -.153 & -.119 & .075 & -.007 & .075 & .135 & .135 & .240 & .108 & .264 & & .141 \\
\hline WtPS & .294 & .234 & .424 & $.554 *$ & $.831 * *$ & $.510 *$ & -.257 & -.194 & .124 & .234 & -.297 & $.674 * *$ & $.998 * *$ & .614 & $-.510^{*}$ & $.528 *$ & . 141 & \\
\hline
\end{tabular}

$*, * *=$ significant at $5 \%$ and $1 \%$ probability levels respectively.

\section{KEY:}

$\mathrm{NOL}=$ number of leaflets per plant, $\mathrm{NOB}=$ number of branches per plant, $\mathrm{PH}=$ plant height, $\mathrm{LL}=$ leaf length, $\mathrm{LW}=$ leaf width

$\mathrm{PDL}=$ peduncle length, DTF= number of days to flower, DTFF = number of days to fifty percent flowering, DTFFRp= number of days to first fruit ripening, $\mathrm{DTM}=$ number of days to maturity, $\mathrm{FPI}=$ fruit per inflorescence, $\mathrm{WPF}=$ weight per fruit, $\mathrm{FL}=$ fruit length, $\mathrm{FD}=$ fruit diameter, $\mathrm{FPP}=$ number of fruit per plant, and FYPP $=$ fruit yield per plant, $1000 \mathrm{SW}=1000$ seed weight and $\mathrm{WtPS}=$ weight per seed 


\section{Discussion}

The low yield obtained for some of the accession used might be due to nondevelopment of flowers into fruits on the plants pedicels. The poor fruit set may be as a result of high diurnal temperatures and relative humidity that are not conducive for good fruit set [OLANIYI \& al. 2010] .The observed differences in growth and yield components of the various accessions suggest that this might be attributed to the differences in ecological distribution as revealed in the various collection locations. Also, the variation in the agronomic and yield components traits analysed is most likely to be due to genetic variability existing among the accessions since they were grown under the same environ-mental conditions.

Genetic diversity can be estimated using measurements of morphological attributes. This is a simple technique for quantifying genetic variation and assessing genotype performance under appropriate growing environments [FUFA \& al. 2005; SHUAIB \& al. 2007]. The precise, fast and reliable identification of important plant varieties is essential in agriculture and plant breeding purposes [WEISING \& al. 2005]. Clustering of accessions used in this study into cherry and classic fruit groups corresponding to varietal types was similar to the results of KWON \& al. 2009, who characterized 63 tomato varieties of Korea using SSR markers and morphological descriptors. Non-significant association between the clustering pattern and geographical origin of these materials is in agreement with the report by $\mathrm{HU} \&$ al. 2012 in their work with 67 argentine tomato varieties. HU \& al. 2012, also reported that fruit shape had the most variable types (seven). The 19 accessions used for this study may be identified as distinct varieties. However, molecular characterization using SSR markers is on-going to ascertain this result.

Highly significant differences among the accessions for all attributes measured is an indication of enough genetic variability and diversity of the accessions hence the scope for improvement of this crop. Similar observations have been reported on 14 characters [SINGH \& RAJ, 2004; HIDAYATULLAH \& al. 2008] in tomato. [MOHAMMED \& al. 2012] also had similar findings of significant differences for all the traits they studied. Moreover, higher values of genotypic and phenotypic variances observed for plant height, number of branches, leaf length, leaf width, number of leaflets, days to flower, days to $50 \%$ flower, days to fruit ripening, fruit per plant, fruit diameter, fruit weight, day to maturity and fruit yield per plant indicate the existence of high magnitude of variability among the accessions with respect these attributes.

Smallest differences observed between PCV and GCV values of attributes such as leaf length, leaf width, days to flower, days to 50\% flower, days to fruit ripening, fruit length, fruit per inflorescence, fruit diameter, fruit weight, days to maturity and 1000 seed weight suggest lesser influence of environmental factors on their expression. Selection for improvement of tomato for these attributes is likely to be most effective. Relatively higher differences between PCV and GCV values recorded for plant height, number of branches, number of leaflets, fruit per plant, peduncle length and fruit yield indicate more influences of environmental factors than other attributes studied.

Very high heritability estimates for leaf length, leaf width, days to flower, days to $50 \%$ flowering, fruit per plant, fruit length, fruit diameter, fruit weight and 1000 seed weight indicate possibility of improvement through selection. Similar results have been reported by TASISA \& al. 2011 and ULLAH \& al. 2012. However, PARNSE, 1957 stated that greater usefulness of considering estimate of genetic advance as an effective selection tool lies in 
accompanied heritability estimates. Hence, very high genetic advance accompanied by high heritability estimates for leaf length, leaf width, days to flower, days to $50 \%$ flowering, fruit per plant, fruit length, fruit diameter, fruit weight and 1000 seed weight suggest simple inheritance system for these traits. FEHMIDA \& AHMED, 2007 reported similar results for plant height, number of fruits per plant, fruit size and weight of 10 tomatoes.

Positive and significant association of fruit yield per plant $(\mathrm{P}<0.05)$ with plant height, number of branches per plant and leaf length shows that taller plants, bearing more branches and longer leaves tend to yield higher as compared to shorter plants. This may be explained by the greater photosynthetic products available for partitioning to fruit production. Positive and significant association of number of fruits per plant with number of fruit per inflorescence is an indication of increased number of fruits with increased number of fruit bearing inflorescence. Weight per fruit which is a function of fruit size had predictably positive and significant association with fruit length and fruit diameter. MOHANTY, 2002 had reported positive and significant correlation of number of fruits per plant with fruit size and single fruit weight. More branching accessions of tomato tend to flower and mature late as shown in the negative and significant association of number of branches per plant with days to flower, days to fruit ripening and days to maturity. This may be due to the fact that much time is spent by the plant in growing more vegetative branches, hence extending its lifespan. Therefore, a breeder interested in improvement for early maturity in tomato may select plants with less number of branches.

\section{Conclusion}

The agronomic and genetic parameters discussed here are functions of the environment, so estimates may differ in other environments as well as agronomic performance. However, based on the high genetic advance accompanied by high heritability estimates for different attributes studied, especially, days to $50 \%$ flowering, fruit per plant, fruit length, fruit diameter, fruit weight and 1000 seed weight we could conclude that the determinant genetic effects of the phenotypic expression of these characters are fundamentally of the additive type. Hence, a high response should be achievable after several selection cycles.

\section{Notes on contributors}

Dickson Junior NWOSU is a Plant Breeder/Geneticist with interest in crop improvement. His bias is towards cowpea improvement especially for insect resistance. He is currently exploring cowpea wild relative as a genepool for insect resistance gene. As a research scientist, he also works as crop conservationist and characterization of several crop species in African.

Christopher NWADIKE is a plant breeder and horticulturist interested in improvement of Phaseolus vulgare and indigenous crop species of sub-Saharan Africa. His is also currently involved in maize improvement Nigeria.

Gloria AFOLAYAN is a plant breeder and research scientist working on sorghum improvement. She is also a conservationist.

Sunday ALADELE is a Plant Breeder and biodiversity conservation expert.

Anthony Ugochukwu OKERE is a Forest Biologist and Silviculturist with a special interest in improvement of indigenous timber, fruit trees, agronomic crops, medicinal plant species, forest 


\section{GENETIC AND AGRONOMIC EVALUATION OF RAINFED TOMATO ...}

biotechnology and biodiversity conservation. His work focuses in the plant genetic resources conservation, seed storage, plant propagation, characterization of mahogany etc.

Peter OMONOMO is a Crop Production Technologist working on several crops including cowpea (cultivated and wild relatives), okra and soybean.

\section{References}

AGARWAL S. \& RAO A. 2000. Tomato lycopene and its role in human health and chronic diseases. CMAJ. 163(6): 739-44.

AKANBI C. \& OLUDEMI F. 2003. Effect of processing and packaging on the lycopene content of tomato products. International Journal of Food Production. 7: 139-151.

EID M. H. 2009. Estimation of heritability and genetic advance of yield traits in wheat (Triticum aestivum L.) under drought conditions. International Journal of Genetics and Molecular Biology. 1(7): 115-120.

FAOSTAT 2010. Food and Agricultural Commodities Production. Available online: http://faostat.fao.org (accessed 21 July 2012).

FEHMIDA A. \& AHMAD S. 2007. Morphological comparisons of three tomato cultivars from Azad Jammu and Kashmir, Pakistan. Sarhad Journal of Agriculture. 23(2): 313-318.

FUFA H., BAENZIGER P., BEECHER I., DWEIKAT V., GRAYBOSCH R. \& ESKRIDGE K. 2005. Comparison of phenotypic and molecular marker-based classifications of hard red winter wheat cultivars. Euphytica. 145: $133-146$.

HIDAYATULLAH S., AHMED J., GHAFOOR A. \& MAHMOOD T. 2008. Path coefficient analysis of yield components in tomato (Lycopersicon esculentum). Pakistan Journal of Botany. 40(2): 627-635.

HU X., WANG H., CHEN J. \& YANG W. 2012. Genetic diversity of Argentina tomato varieties revealed by morphological traits, simple sequence repeat, and single nucleotide polymorphism markers. Pakistan Journal of Botany. 44(2): 485-492.

IBPGR. 1997. Descriptors for tomato (Lycopersicom spp.). International Board for Plant Genetic Resources. Rome, Italy, pp. 24-36.

JOHNSON H., ROBINSON H. \& COMSTOCK R. 1955. Estimates of genetic and environmental variability in soyabean. Agronomy Journal. 47: 314-318.

KANYOMEKA L. \& SHIVUTE B. 2005. Influence of pruning on tomato production under controlled environments. Agricultura Tropica et Subtropica. 32(2): 79-81.

KWON Y., PARK S. \& YI S. 2009. Assessment of genetic variation among commercial tomato (Solanum lycopersicum L.) varieties using SSR markers and morphological characteristics. Gene and Genomics. 31(1): 1-10.

MOHANTY B. 2002. Studies on variability, heritability inter relationship and path analysis in tomato. Annals of Agricultural Research. 2(1): 65-69.

MOHAMMED S., ALI E. \& MOHAMED T. 2012. Study of heritability and genetic variability among different plant and fruit characters of tomato (Solanum lycopersicon L.). International Journal of Scientific \& Technology Research. 1(2): 55-58.

National Centre for Genetic Resources and Biotechnology (NACGRAB) and National Agriculture Seed Council (NASC). 2013. Catalogue of crop varieties released and registered in Nigeria. Volume 5. Iowa State University, USA.

OLANIYI J., AKANI W., ADEJUMO T. \& O AKANDE. 2010. Growth, Fruit Yield and Nutritional Quality of Tomato Varieties. African Journal of Food Science. 4(6): 398-402, June 2010. (C2010 Academic Journals. Available online http://www.academicjournals.org/ajfs .ISSN 1996-0794.

PARNSE M. 1957. Genetics of quantitative characters in relation to plant breeding. Indian Journal of Genetics. 17: 318-328.

PBTOOLS, version 1.3. 2014. Biometrics and Breeding Informatics, PBGB Division, International Rice Research Institute, Los Baños, Laguna.

SAS Institute. 2002. Statistical systems version 9. SAS Institute Inc. Cary, NC27513, USA.

SAINJU M., DRIS R. \& SINGH B. 2003. Mineral nutrition of tomato. Food Agriculture \& Environment. 1(2): 176-183.

SHUAIB M., ALAM Z., ZAHIR A., WAQAR A., TAUFIQ A. \& IKHTIAR K. 2007. Characterization of wheat varieties by seed storage protein electrophoresis. African Journal of Biotechnology. 6: 497-500.

SINGH A. \& RAJ N. 2004. Variability studies in tomato under cold arid condition of Ladakh. Horticulture Journal. 17: 67-72.

SINGH R. \& CHAUDHARY B. 1985. Biometrical methods in quantitative analysis. Kaljuni Publishers, New Delhi, India. 
SNEDECOR G. \& COCHRAN W. 1967. Statistical Methods, 6th ed., Iowa State University Press. USA.

SONGSRI P., JOGLlOY S., KESMALA T., VORASOOT N., AKKASAENG C. \& HOLBROOK C. 2008. Heritability of drought resistant traits and correlation of drought resistance and agronomic traits in peanut. Crop Science. 48: 2245-2253.

TASISA J., BELEW D., BANTTE K. \& GEBRESELASSIE W. 2011. Variability, heritability and genetic advance in tomato (Lycopersicon esculentum Mill.) genotypes in West Shoa, Ethiopia. American-Eurasian Journal of Agriculture \& Environmental Science. 11(1): 87-94.

TAZEEN M. NADIA K. \& FARZANA N. 2009. Heritability, phenotypic correlation and path coefficient studies for some agronomic characters in synthetic elite lines of wheat. Journal of Food, Agriculture and Environment. 7(3-4): 278-282.

ULLAH M., HASAN M., CHOWDHURY A., SAKI A. \& RAHMAN A. 2012. Genetic variability and correlation in exotic cucumber (Cucumis sativus L.) varieties. Bangladesh Journal of Plant Breeding and Genetics. 25(1): 17-23.

WEISING K., NYBOM H., WOLFF K. \& KAHL G. 2005. Application of DNA finger printing in plant sciences. In: DNA fingerprinting in plants: Principles, Methods and Applications, CRC Press, Boca Raton, pp. 235-276.

How to cite this article:

NWOSU D. J., NWADIKE C., AFOLAYAN G., ALADELE S., OKERE A. U. \& OMONOMO P. 2018. Genetic and agronomic evaluation of rainfed tomato (Solanum lycopersicum L.) accessions in Ibadan, Nigeria. J. Plant Develop. 25: 43-57. https://doi.org/10.33628/jpd.2018.25.1.43 